\title{
Point of Care Testing for the Diagnosis of Fungal Infections: Are We There Yet?
}

\author{
Juergen Prattes ${ }^{1,2} \cdot$ Sven Heldt $^{3} \cdot$ Susanne Eigl $^{3} \cdot$ Martin Hoenigl $^{1,2,3,4}$
}

Published online: 7 April 2016

(C) The Author(s) 2016. This article is published with open access at Springerlink.com

\begin{abstract}
Diagnostic tools for invasive fungal infections have continuously improved within the last decades. Nowadays, cultural methods, antigen testing, and molecular tests, such as polymerase chain reaction, are widely used. These methods, however, are accompanied with different limitations as various availability, various turnaround time or high costs. A new generation of point-of-care test has shown promising results in various studies and may overcome some of these limitations. We therefore reviewed the literature for the most promising new point-of-care tests for invasive aspergillosis (Aspergillus-specific lateral-flow device test, Aspergillus proximity ligation antigen assay), cryptococcosis (cryptococcal lateral-flow assay), and for histoplasmosis (loop-mediated isothermal amplification assay).
\end{abstract}

Keywords Point-of-care $\cdot$ Diagnosis $\cdot$ Aspergillus · Histoplasma · Cryptococcus $\cdot$ Lateral-flow device $\cdot$ Invasive fungal infections $\cdot$ Diagnostic tools $\cdot$ Fungal infections . Review

This article is part of the Topical Collection on Clinical Mycology Lab Issues

Juergen Prattes

juergen.prattes@medunigraz.at

$\triangle$ Martin Hoenigl

martin.hoenigl@medunigraz.at

1 Section of Infectious Diseases and Tropical Medicine, Medical University of Graz, Auenbruggerplatz 15, 8036 Graz, Austria

2 CBmed - Center for Biomarker Research in Medicine, Graz, Austria

3 Division of Pulmonology, Medical University of Graz, Graz, Austria

4 Division of Infectious Diseases, Department of Medicine, University of California-San Diego, San Diego, CA, USA

\section{Introduction}

Invasive fungal infections (IFIs) are a major cause of morbidity and mortality in immunocompromised patients [1-3]. Host factors such as severe and prolonged neutropenia, allogeneic stem cell transplantation, prolonged use of corticosteroids, prolonged hospitalization at an intensive care unit (ICU), and receipt of recognized $\mathrm{T}$ cell immunosuppressants may predispose patients for developing IFI $[1,4]$.

IFIs have a wide spectrum of clinical presentations, and diagnoses mostly rely on laboratory-based results. Culturebased methods are valuable but limited by time to results, and due to the relatively insensitive for the detection of fungal pathogens $[5,6]$. Matrix-assisted laser desorption/ionization time-of-flight mass spectrometry (MALDI-TOF MS) is a very promising development for identification of culture isolates. MALDI-TOF testing is simple to perform, accurate, can technically identify nearly every organism, and may even detect resistance [5]. Nevertheless, impact of MALDI-TOF testing on early diagnosis and treatment of IFIs is limited, as a positive culture result is a prerequisite for that method. Currently, amplification-based assays are under development that may ultimately replace culture-based tests in the laboratory [5]. Amplification-based assays may provide results to the laboratory within $20 \mathrm{~min}$ to $5 \mathrm{~h}$. New multiplex polymerase chain reactions (PCRs) are often able to detect a selection of multiple pathogens in a single session [5]. However, currently available amplification assays are mostly limited by relatively high costs, inconsistent performance in terms of sensitivities and specificities, and lack of standardization. Loop-mediated isothermal amplification (LAMP) is a cheaper and more simple technique than traditional PCR, and therefore an advantageous alternative, providing results to the laboratory in less than $60 \mathrm{~min}$ [5]. Thus, LAMP for diagnosis of histoplasmosis will be discussed in this review. 
Besides molecular diagnostic tools, antigen-based tests are widely used in clinical routine for IFI diagnosis. They are much faster compared to culture and able to provide quantitative results to the clinician optimally within a day [5]. Depending on the setting, however, time to results may vary, depending on factors such as transport to the laboratory that performs the test. In contrast, qualitative antigen-based assays may allow point-of-care (POC) diagnosis of IFI within minutes, frankly on the bedside. Antigen-based POC tests are currently available for Aspergillus spp. and Cryptococcus spp. [5], and both will be discussed in this review.

\section{POC Diagnosis of Invasive Aspergillosis}

Aspergillus spp. and other molds are the major cause of IFIs among patients with underlying hematological malignancies $[3,7]$. Successful management of invasive aspergillosis (IA) in these critically ill patients requires early and reliable diagnosis and rapid initiation of appropriate antifungal therapy. A study analyzing autopsy data over two decades has shown in 2013 that rates of premortem diagnosis of IA have increased over the last decade. While $84 \%$ of the IFIs were diagnosed postmortem in the first 5 years of the study, the rate decreased to $49 \%$ in the last 4 years of the study [8]. Most likely reasons for an ongoing increase of premortem IA diagnoses are the introduction of antigen testing in serum and bronchoalveolar lavage fluid (BALF) specimens [9, 10]. Galactomannan antigen (GM) testing is currently considered the gold standard when it comes to biomarkers for IA diagnosis. Several studies have evaluated the performance of GM detection in different specimen (BALF, blood, urine, cerebrospinal fluid) and proposed its utility in daily clinical routine. GM determination, which is performed using the Platelia ${ }^{\text {тм }}$ Aspergillus enzyme immunoassay (Bio-Rad, France), however, has some limitations. First, the variable turnaround time within different centers depending on the amount of specimen sent in for GM determination. The Platelia ${ }^{\mathrm{TM}}$ Aspergillus enzyme immunoassay (BioRad, France), approved from the FDA as an adjunctive test for IA diagnosis, is performed on a 96-well plate. Second, GM determination requires specially equipped laboratories and trained staff that is not available in all centers. Third, the GM ELISA also detects antigens produced by Geotrichum capitatum and cross-reacts with other opportunistic fungal pathogens like Histoplasma spp. and may with Cryptococcus neoformans [11-13]. Qualitative POC assays for GM detection are currently in development and may be promising for the future, once developed, evaluated, and approved.

\section{Aspergillus-Specific Lateral-Flow Device Test}

The POC Aspergillus-specific lateral-flow device (LFD) test has been developed more than 5 years ago and evaluated in a number of studies. The LFD is an immunochromatographic assay using a mouse monoclonal antibody, JF5, which binds to an extracellular glycoprotein antigen from Aspergillus spp., only secreted during active growth. Minimal required training, simple handling by using BALF samples without any pretreatment, no need for specially equipped laboratories, rapid availability of test results within $15 \mathrm{~min}$, and low costs are the major advantages of the LFD. In case of serum testing, samples need to be pretreated by heating, centrifugation, and adding a buffer solution according to the manufacturer's recommendations $[14,15]$. Results are read by eye after 15-min incubation time and are interpreted depending on the intensity of the test line as negative $(-)$ or weak $(+)$ to strong $(+++)$ positive. Even though the results are read by the naked eye and one may argue that this may lead to poor reproducibility, Wiederhold and colleagues performed a study using a guinea pig model of IA and tested serum and BALF samples in two independent laboratories. Thirty-two of 33 (97\%) serum samples and 26/33 (79 \%) BALF samples were in agreement within these two laboratories, indicating a good reproducibility [16]. Cross-reactivities are also rare with the LFD. Only cross-reactions with Penicillium spp. are described in the original publication [15]. Since these data were published, interest in this new POC test has increased and several clinical studies evaluated the clinical performance of the LFD test in various patient cohorts and different specimen.

In one of the first in vivo studies evaluating BALF samples from 29 patients with hematological malignancies, the LFD yielded sensitivity of $100 \%$ and a specificity of $81.8 \%$ for diagnosis of IA. Four samples yielded false-positive results, but all of them were interpreted as weak positive only [17]. A further study on BALF-LFD in hematological patients also yielded a $100 \%$ sensitivity and a specificity of $83 \%$ [18]. Even though this study was limited by a very small sample size of hematological patients $(n=7)$, results were similar to the prior mentioned study. The largest study evaluating the performance of the BALF-LFD test in hematological malignancy patients was published in 2015, and 95 BALF samples were analyzed from 72 prospectively enrolled patients of whom 27 patients (30 samples) had probable IA [19]. Per patient sensitivity was lower compared to the former studies (71\%), but specificity was similar (76\%). This may partly explained due to the fact that all patients with false-negative results within the last study were on antifungal prophylaxis/treatment at the time of bronchoscopy. Mold active prophylaxis/treatment may cause a significant decrease of BALF-LFD sensitivity as shown in a recently published study. This retrospective analysis yielded BALF-LFD sensitivity of $86 \%$ in patients not receiving mold-active agents versus $52 \%$ in patients receiving moldactive agents $(p=0.006)$ [20]. In addition, in most studies performed on BALF-LFD, no or little information is given on pretreatment of BALF samples prior to testing. This, however, is of particular interest as pretreatment with dithiothreitol (a 
commonly used mucolytic agent for respiratory tract samples) containing liquefying agents significantly alters LFD test line intensities and reduces GM levels [21]. In another recently published study by Johnson et al., the performance of realtime PCR, LFD, and GM in BALF samples obtained from patients at risk for IA was evaluated and showed that PCR and LFD had perfect sensitivity of $100 \%$ compared to $87.5 \%$ sensitivity for GM detection [22]. Specificity was slightly lower for both, PCR and LFD, when calculated for each test ( 87 and $80 \%$, respectively) but superior to specificity of GM detection with a specificity $66.6 \%$. PCR and LFD results showed a nearly perfect agreement with a kappa coefficient value of 0.93 . Thus, combination of PCR and LFD did not result in an increase of specificity $(85.7 \%$ ), showing the high potential of the LFD test.

Similar to GM, the LFD may also be performed in serum samples. Results from studies investigating the performance of the LFD when using serum samples, however, showed less promising results compared to BALF testing. This may be in part explained due to the fact that systemic antifungal prophylaxes/therapy may have a stronger influence on serum samples compared to BALF samples [23] which was also shown for GM detection [24]. Held and colleagues reported a sensitivity and specificity of about 40 and $86.8 \%$ for serumLFD testing in hematological stem cell transplant (HSCT) recipients when only one positive LFD was required for IA diagnoses and a sensitivity of only $20 \%$ and specificity of $97.8 \%$ when two consecutive positive LFD samples were required for diagnosis [25]. Positive samples, however, need to be interpreted in context with clinical signs and symptoms and should trigger further investigations as demonstrated by White and colleagues, who reported a poor positive predictive value (PPV) of $67 \%$ when using serum-LFD results for IA diagnosis but a remarkable increase of the PPV when using LFD in combination with either PCR (100\%) or GM (80\%) for diagnosis [26].

As seen with GM, BALF samples seem to be the most promising when using the LFD test for IA diagnosis in nonneutropenic patients, in particular patient collectives outside of the hematological malignancy setting. A number of studies on BALF-LFD performance in patient cohorts besides hemato-oncological patients have been published, revealing consistent and promising results of the test. In solid organ transplant (SOT) recipients, for example, sensitivities between 91 and $100 \%$ as well as specificities of $80-83 \%$ were reported $[17,27]$. Similar performance could be observed for ICU patients with sensitivity of $80 \%$ and specificity of $81 \%$ [28]. The high negative predictive value of $96 \%$ in this study may be used in clinical routine to rapidly rule out suspected IA in ICU patients and may withhold antifungal therapy. This is of particular interest in ICU patients as up to two thirds of ICU patients receiving antifungal agents without evidence for IFIs [29]. Performance of BALF-LFD was also evaluated in patients with underlying pulmonary disease [6]. Sensitivity for IA diagnosis reached $77 \%$ and specificity $92 \%$. Thus, the LFD showed a significant higher sensitivity compared to mycological culture (77 vs $29 \%$ ) and even a higher specificity compared to BALF-GM determination (92 vs $81 \%$ ). In contrast, data on the performance of serum LFD testing in nonneutropenic patients, like ICU patients or SOT recipients, are lacking to date.

In conclusion, the LFD test seems to be a promising POC test for IA diagnosis as it showed good performance in clinical studies and overcomes some of the limitations given with GM determination as variable turnaround time, availability, or cross-reactions. Performances of the LFD test in the different patient cohorts as well as in different specimen (using published studies) are displayed in Tables 1 and 2.

\section{Proximity Ligation Assay as a Diagnostic Technique for Invasive Aspergillosis}

Proximity ligation assays (PLA) are very specific antibodybased tests with high sensitivity and therefore a valuable diagnostic option. Johnson et al. [30] successfully designed a PLA for detecting a Aspergillus mannoprotein. They used the monoclonal antibody JF5, which is the same antibody used with the Aspergillus LFD test, for targeting a Aspergillus-specific extracellular mannoprotein. In their study, Aspergillus culture filtrate was gained and spiked to saline and serum [30]. PLA, GM detection by using the Platelia ${ }^{\mathrm{TM}}$ Aspergillus enzyme immunoassay (Bio-Rad, France), and the LFD were performed with these samples. The results of the PLA showed a 10- to 100-fold higher sensitivity compared to the GM assay and a 1000-fold higher sensitivity compared to the LFD assay. Furthermore, three BALF samples were tested with the PLA in cases with probable IA and positive GM assay, LFD, and PCR. The PLA results were coherently positive in all three cases [30]. In addition, the PLA assay also had a high specificity and showed no cross-reactivity when tested with culture filtrates of other fungal species (i.e., Candida, Mucor, Fusarium). In conclusion, the Aspergillus PLA developed by Johnson et al. seems to be highly sensitive and specific and warrants future investigation in a higher number of clinical samples.

\section{POC Diagnosis of Cryptococcosis}

Cryptococcal meningitis is a life-threatening opportunistic fungal infection caused by Cryptococcus spp., primarily by the pathogenic species Cryptococcus neoformans var. grubii and var. neoformans and Cryptococcus gattii. Cryptococcosis occurs mostly HIV related [31], but increasing incidence has also been reported in SOT recipients. Overall, cryptococcal meningitis is estimated to affect nearly a million patients per year, 
Table 1 Aspergillus LFD performance in BALF and serum in various patient cohorts

\begin{tabular}{lllllll}
\hline Study & Risk group & $\begin{array}{l}\text { Sample size } \\
(n \text { of patients })\end{array}$ & Specimen & Sensitivity & Specificity & Reference \\
\hline Hoenigl 2012 & HM & 29 & BALF & 100 & 81.8 & {$[17]$} \\
Miceli 2015 & HM & 7 & BALF & 100 & 83 & {$[18]$} \\
Prattes 2015 & HM & 72 & BALF & 71 & 76 & {$[19]$} \\
Johnson 2015 & HM and non-HM & 32 & BALF & 100 & 80 & {$[22]$} \\
Hoenigl 2012 & SOT & 10 & BALF & 100 & 80 & {$[17]$} \\
Willinger 2014 & SOT & 47 & BALF & 91 & 83 & {$[27]$} \\
Eigl 2015 & ICU & 133 & BALF & 80 & 81 & {$[28]$} \\
Prattes 2014 & Respiratory & 221 & BALF & 77 & 92 & {$[6]$} \\
Held 2013 & Disease & 101 & Serum & $40^{\mathrm{a}}$ & $86.8^{\mathrm{a}}$ & {$[25]$} \\
HSCT & & & $20^{\mathrm{b}}$ & $97.8^{\mathrm{b}}$ & \\
White 2013 & HM & 103 & Serum & $81.8^{\mathrm{a}}$ & $84.8^{\mathrm{a}}$ & {$[26]$} \\
\hline
\end{tabular}

$H M$ hematological patients, SOT solid organ transplant recipients, $I C U$ intensive care unit, $H S C T$ hematological stem cell transplantation recipients, $B A L F$ bronchoalveolar lavage fluid

${ }^{\mathrm{a}}$ Single testing $=$ a minimum of one positive LFD results is required for diagnosis

${ }^{\mathrm{b}}$ Multiple testing $=\mathrm{a}$ minimum of two or more positive LFD results are required for diagnosis with more than 600,000 deaths predominantly in developing resource-limited countries [32]. To reduce the high mortality rate, which reaches $12 \%$ in industrialized countries like the USA [33] and almost $90 \%$ in sub-Saharan and South Africa $[34,35]$, rapid and reliable diagnostic tools for detection of the basidiomycetous fungi are of utmost importance. Mycological culture is still considered the gold standard but is limited long turnaround time. In addition, appropriately equipped laboratories as well as technical expertise are required for culturing cerebrospinal fluid (CSF), which both are often not available in resource-limited countries. Hence, diagnosis of cryptococcosis mostly relied on direct microscopy of CSF, though this is characterized by poor sensitivity. Therefore, detection of cryptococcal antigen $(\mathrm{CrAg})$, a component of the cryptococcal polysaccharide capsule glucoronoxymannan (GXM), has become increasingly important. To date, two main methods for detection of the $\mathrm{CrAg}$ exist. On the one hand, latex agglutination (LA) and on the other hand the enzyme immunoassay (EIA). Both methods are highly sensitive and specific, and less time-intensive compared to culture or microscopy [36]. However, LA as well as EIA have limitations. First, they require laboratory infrastructure and skilled technicians and second, false-negative results and prozone effects (= falsenegative results due to very high concentrations of particular analyte in immunoassays) were well known [37].

\section{Cryptococcal Antigen Lateral-Flow Assay}

The CrAg lateral-flow assay (LFA) is a POC test for the detection of $\mathrm{CrAg}$, which was developed by Immy Inc. (Norman, OK) in 2009. The CrAg LFA satisfies all requirements for a POC test: low costs (approximately $2 \$$ per strip in resource limited settings), excellent test performance, easy to use and rapid test results (available in $10 \mathrm{~min}$ ) [38]. Moreover, the CrAg LFA is temperature stable and cross-reactions with other fungi are rare. For testing, $40 \mu \mathrm{l}$ of body fluid (serum, plasma, urine, and CSF) without pretreatment are applied to a reservoir of the LFA test strip. If GXM is present, the anti-GXM monoclonal antibodies - the test uses two monoclonal antibodies and can recognize all four GXM serotypes (A-D) - forms a visible line. The handling of the test and the interpretation of the test line result have been well demonstrated in publications $[39,40]$. In one study, sensitivity of the serum CrAg LFA of $100 \%$ and a specificity of $99.8 \%$ was reported, when using
Table 2 Performance of the BALF Aspergillus LFD for probable/proven invasive pulmonary aspergillosis versus no evidence for invasive pulmonary aspergillosis (per BALF sample) in different patient cohorts

\begin{tabular}{lllll}
\hline Patient group & Sensitivity & Specificity & PPV & NPV \\
\hline Solid organ transplantation & $94 \%(15 / 16)$ & $92 \%(89 / 97)$ & $65 \%(15 / 23)$ & $99 \%(89 / 90)$ \\
Intensive care unit & $79 \%(26 / 33)$ & $85 \%(176 / 206)$ & $57 \%(26 / 46)$ & $96 \%(176 / 183)$ \\
Respiratory diseases & $77 \%(24 / 31)$ & $92 \%(195 / 211)$ & $60 \%(24 / 40)$ & $97 \%(195 / 202)$ \\
Hematological malignancies & $65 \%(30 / 47)$ & $89 \%(88 / 99)$ & $73 \%(30 / 41)$ & $84 \%(88 / 105)$ \\
\hline
\end{tabular}

Data derived from published studies: $[6,14,17-20,27,28,66]$

$P P V$ positive predictive value, $N P V$ negative predictive value 
serum LA test as gold standard [41]. In the same year, another study also reported a $100 \%$ sensitivity of the serum CrAg LFA which was higher than the $91 \%$ found for serum LA. Importantly, this study included only very few patients with HIV infection, indicating the potential diagnostic value of the CrAg LFA also for other populations with cryptococcal disease [42]. Other studies comparing the CrAg LFA to other $\mathrm{CrAg}$ based tests in low-income countries show similar results: sensitivities of 95.6-100\% and specificities of 96.9-99.5\% when using serum specimens $[43,44]$, and sensitivities of $80-100 \%$ and specificities of 73.8-91.5\% when using urine samples (also depending on the diluents) [45]. The reliable performance of the CrAg LFA was confirmed in studies evaluating its performance compared to culture or composite reference standard: sensitivities of $98.3-100 \%$ in serum samples, $92-98 \%$ in urine specimens, and 86.1-100\% in CSF samples [44, 46-48]. Since 2011, the World Health Organization (WHO) has added the CrAg LFA to the LA test as preferred method for diagnosis of cryptococcal disease [49].

To summarize, the CrAg LFA has several advantages: multiple samples can be run simultaneously, cost-effective, and do not need electricity, specially equipped laboratory, or skilled technicians. Importantly, CrAg LFA is not useful to check treatment response, as the clearance of $\mathrm{CrAg}$ is a slow and also independent process that devitalize the yeast $[50,51]$. During effective antifungal therapy, CrAg LFA titers may therefore remain elevated $[52,53]$.

CrAg has been confirmed as a reliable predictor of development of cryptococcal disease after initiating antiretroviral therapy (ART), and even mortality [47, 54-56]. Twenty to $30 \%$ of patients in resource-poor countries present cryptococcal meningitis within 3 months after initiating ART [57, 58]. French et al. reported that $\mathrm{CrAg}$ may be detectable as early as 100 days before the beginning of symptomatic cryptococcal disease [59]. Therefore, the WHO recommends routine serum or plasma $\mathrm{CrAg}$ screening in HIV-positive patients without ART and a CD4 cell count $<100$ cells/ $\mathrm{mm}^{3}$ [49]. With regard to the role of the test in preemptive screening, one important questions remains to be answered: how to proceed with patients that do not have symptoms of cryptococcal meningitis but a positive CrAg result? While the WHO recommends a "screen and treat asymptomatic positives with fluconazole" strategy, future studies are needed to answer this question.

In conclusion, the diagnostic accuracy of $\mathrm{CrAg}$ has been evaluated in multiple studies in resource-rich and resourcelimited countries and showed excellent sensitivity and specificity on serum, plasma, urine [44, 48], and CSF samples [47]. A positive CrAg allows the treating physician to initiate appropriate antifungal therapy without delay and may additionally predict mortality risk. CrAg LFA may also play a future role in preemptive screening, consequently in cost reduction and outcome improvement [45, 60-62]. This POC test, therefore, clearly has the potential to markedly improve the early diagnosis of cryptococcosis.

\section{Advancement in the Diagnosis of Histoplasmosis}

Histoplasmosis and especially progressive disseminated histoplasmosis $(\mathrm{PDH})$ in patients with immunodeficiency is caused by Histoplasma capsulatum, a fungus endemic in North and South America, Africa, Asia, and Australia [63]. Diagnosis is challenging; in particular, the current alternatives to culture diagnostics are too expensive for resourcechallenged countries [64]. Rapid and reliable assays are important for the treatment of PDH, as mortality rate increases up to $42 \%$ when therapy is delayed and up to $95 \%$ when PDH is misdiagnosed [64]. The diagnostic process is also complicated as symptoms of PDH are unspecific and similar to infections with mycobacteria or leishmania [65]. The currently available diagnostics for $H$. capsulatum comprise detection by culture, which can be enhanced by culture identification with the AccuProbe $^{\circledR}$ test. The main problem with this method is that culturing $H$. capsulatum can take a few weeks, and the AccuProbe ${ }^{\circledR}$ test is expensive [64]. As an alternative, there are different PCR-based assays available. However, these are also expensive and not well evaluated yet [65].

Scheel et al. [64] conducted a pilot study in which they developed a LAMP assay and evaluated its sensitivity and specificity in comparison to the PCR-based assays. LAMP is an alternative method for DNA amplification using a different polymerase (Bst) as used for traditional PCR (Taq). Bst-polymerase is cheaper and more robust than Taq. LAMP also requires less expensive equipment compared to traditional PCR. In their study, Scheel et al. [64] chose the Hcp 100-locus of $H$. capsulatum for amplification and designed a LAMP primer, as that locus has only few similarities to related microorganisms, and there are no any known mutations that may lead to false negativity. Furthermore, they collected different geographic subspecies of $H$. capsulatum isolates $(n=91)$, extracted the DNA, and proceeded with PCR and LAMP to compare the sensitivities of these two assays, and to evaluate the limit of necessary genomes for the LAMP assay to become positive (limit of detection (LOD)). In addition, Scheel et al. [64] collected urine samples from healthy persons $(n=10)$ as well as from HIV patients with PDH $(n=6$; proven by clinical symptoms, positive antigen detection and positive culture with urine samples), where they compared sensitivity and specificity of PCR and LAMP. The LOD was noted to be a median of 6 genomes (from 1 to 30 genomes, strain-dependent), and 10fold lower than the LOD of PCR. Within an incubation time of $1.5 \mathrm{~h}$, no cross-reactivity could be recognized when the designed LAMP primer had been used for assays with other fungi, M. tuberculosis or human DNA, which means a specificity of $100 \%$ in this pilot study. Coherent to these findings, there was no false-positive LAMP assay when testing the urine 
samples of the healthy persons. LAMP was only able to detect H. capsulatum DNA in four of the six urine samples (67\%), which was still superior to the simultaneously conducted PCR assays, and was not able to show any positive test result when testing the urine samples. In conclusion, Scheel et al. [64] showed that $H$. capsulatum DNA can be detected from cultured isolates as well as from urine samples of patients with PDH when using their LAMP assay. As the search for a POC test for $H$. capsulatum continues, the study presents interesting findings that may advance diagnosis in the meantime.

\section{Conclusion}

Important advances have been made during the last years in particular with regard to POC diagnosis of cryptococcosis but also for IA. The CrAg LFD not only has excellent sensitivity over all four serotypes of Cryptococcus but is also FDA approved and commercially available; commercial availability of the Aspergillus LFD is still pending. While LAMP may offer significant advancement in the diagnosis of histoplasmosis, the search for reliable POC tests for other fungi continues.

Acknowledgments Open access funding provided by Medical University of Graz.

\section{Compliance with Ethical Standards}

Conflict of Interest Juergen Prattes, Sven Heldt, and Susanne Eigl declare that they have no conflict of interest.

Martin Hoenigl reports grants from Gilead, Merck, and Pfizer outside the submitted work.

Human and Animal Rights and Informed Consent This article does not contain any studies with human or animal subjects performed by any of the authors.

Open Access This article is distributed under the terms of the Creative Commons Attribution 4.0 International License (http:// creativecommons.org/licenses/by/4.0/), which permits unrestricted use, distribution, and reproduction in any medium, provided you give appropriate credit to the original author(s) and the source, provide a link to the Creative Commons license, and indicate if changes were made.

\section{References}

1. Eggimann P, Garbino J, Pittet D. Epidemiology of Candida species infections in critically ill non-immunosuppressed patients. Lancet Infect Dis. 2003;3(11):685-702.

2. Hoenigl M, Zollner-Schwetz I, Sill H, Linkesch W, Lass-Florl C, Schnedl WJ, et al. Epidemiology of invasive fungal infections and rationale for antifungal therapy in patients with haematological malignancies. Mycoses. 2011;54(5):454-9. doi:10.1111/j.1439-0507. 2010.01881.x.

3. Kontoyiannis DP, Marr KA, Park BJ, Alexander BD, Anaissie EJ, Walsh TJ, et al. Prospective surveillance for invasive fungal infections in hematopoietic stem cell transplant recipients, 20012006: overview of the Transplant-Associated Infection Surveillance Network (TRANSNET) Database. Clin Infect Dis. 2010;50(8):1091-100. doi:10.1086/651263.

4. Hoenigl M, Strenger V, Buzina W, Valentin T, Koidl C, Wolfler A, et al. European Organization for the Research and Treatment of Cancer/Mycoses Study Group (EORTC/MSG) host factors and invasive fungal infections in patients with haematological malignancies. J Antimicrob Chemother. 2012;67(8):2029-33. doi:10.1093/ jac/dks155.

5. Caliendo AM, Gilbert DN, Ginocchio CC, Hanson KE, May L, Quinn TC, et al. Better tests, better care: improved diagnostics for infectious diseases. Clin Infect Dis. 2013;57 Suppl 3:S139-70. doi: 10.1093/cid/cit578.

6. Prattes J, Flick H, Pruller F, Koidl C, Raggam RB, Palfner M, et al. Novel tests for diagnosis of invasive aspergillosis in patients with underlying respiratory diseases. Am J Respir Crit Care Med. 2014;190(8):922-9. doi:10.1164/rccm.201407-1275OC.

7. Auberger J, Lass-Florl C, Ulmer H, Nogler-Semenitz E, Clausen J, Gunsilius E, et al. Significant alterations in the epidemiology and treatment outcome of invasive fungal infections in patients with hematological malignancies. Int J Hematol. 2008;88(5):508-15. doi:10.1007/s12185-008-0184-2.

8. Lewis RE, Cahyame-Zuniga L, Leventakos K, Chamilos G, BenAmi R, Tamboli P, et al. Epidemiology and sites of involvement of invasive fungal infections in patients with haematological malignancies: a 20-year autopsy study. Mycoses. 2013;56(6):638-45. doi:10.1111/myc. 12081.

9. Maertens J, Maertens V, Theunissen K, Meersseman W, Meersseman P, Meers S, et al. Bronchoalveolar lavage fluid galactomannan for the diagnosis of invasive pulmonary aspergillosis in patients with hematologic diseases. Clin Infect Dis. 2009;49(11):1688-93. doi:10.1086/647935.

10. Seeber K, Duettmann W, Krause R, Hoenigl M. Usefulness of the serum galactomannan assay for early response assessment and treatment stratifications of invasive aspergillosis. Curr Fungal Infect Rep. 2012;6(3):198-205. doi:10.1007/s12281-012-0099-5.

11. Dalle F, Charles PE, Blanc K, Caillot D, Chavanet P, Dromer F, et al. Cryptococcus neoformans Galactoxylomannan contains an epitope(s) that is cross-reactive with Aspergillus Galactomannan. J Clin Microbiol. 2005;43(6):2929-31. doi:10.1128/JCM.43.6. 2929-2931.2005.

12. Giacchino M, Chiapello N, Bezzio S, Fagioli F, Saracco P, Alfarano $\mathrm{A}$, et al. Aspergillus galactomannan enzyme-linked immunosorbent assay cross-reactivity caused by invasive Geotrichum capitatum. J Clin Microbiol. 2006;44(9):3432-4. doi:10.1128/JCM.00856-06.

13. Vergidis P, Walker RC, Kaul DR, Kauffman CA, Freifeld AG, Slagle DC, et al. False-positive Aspergillus galactomannan assay in solid organ transplant recipients with histoplasmosis. Transpl Infect Dis. 2012;14(2):213-7. doi:10.1111/j.1399-3062.2011. 00675.x.

14. Thornton C, Johnson G, Agrawal S. Detection of invasive pulmonary aspergillosis in haematological malignancy patients by using lateral-flow technology. J Vis Exp. 2012;(61). doi: 10.3791/3721.

15. Thornton CR. Development of an immunochromatographic lateral-flow device for rapid serodiagnosis of invasive aspergillosis. Clin Vaccine Immunol. 2008;15(7):1095-105. doi:10.1128/ CVI.00068-08.

16. Wiederhold NP, Najvar LK, Bocanegra R, Kirkpatrick WR, Patterson TF, Thornton CR. Interlaboratory and interstudy reproducibility of a novel lateral-flow device and influence of antifungal therapy on detection of invasive pulmonary aspergillosis. J Clin Microbiol. 2013;51(2):459-65. doi:10.1128/JCM.02142-12.

17. Hoenigl M, Koidl C, Duettmann W, Seeber K, Wagner J, Buzina W, et al. Bronchoalveolar lavage lateral-flow device test for invasive pulmonary aspergillosis diagnosis in haematological malignancy 
and solid organ transplant patients. J Infect. 2012;65(6):588-91. doi:10.1016/j.jinf.2012.10.003.

18. Miceli MH, Goggins MI, Chander P, Sekaran AK, Kizy AE, Samuel L, et al. Performance of lateral flow device and galactomannan for the detection of Aspergillus species in bronchoalveolar fluid of patients at risk for invasive pulmonary aspergillosis. Mycoses. 2015;58(6):368-74. doi:10.1111/myc.12327.

19. Prattes J, Lackner M, Eigl S, Reischies F, Raggam RB, Koidl C, et al. Diagnostic accuracy of the Aspergillus-specific bronchoalveolar lavage lateral-flow assay in haematological malignancy patients. Mycoses. 2015;58(8):461-9. doi:10.1111/myc.12343.

20. Eigl S, Prattes J, Reinwald M, Thornton CR, Reischies F, Spiess B, et al. Influence of mould-active antifungal treatment on the performance of the Aspergillus-specific bronchoalveolar lavage fluid lateral-flow device test. Int J Antimicrob Agents. 2015;46(4):4015. doi:10.1016/j.ijantimicag.2015.05.017.

21. Prattes J, Koidl C, Eigl S, Krause R, Hoenigl M. Bronchoalveolar lavage fluid sample pretreatment with Sputasol significantly reduces galactomannan levels. J Infect. 2014. doi:10.1016/j.jinf. 2014.11.005.

22. Johnson GL, Sarker SJ, Nannini F, Ferrini A, Taylor E, Lass-Florl $\mathrm{C}$, et al. Aspergillus-specific lateral-flow device and real-time PCR testing of bronchoalveolar lavage fluid: a combination biomarker approach for clinical diagnosis of invasive pulmonary aspergillosis. J Clin Microbiol. 2015;53(7):2103-8. doi:10.1128/JCM.00110-15.

23. Wiederhold NP, Thornton CR, Najvar LK, Kirkpatrick WR, Bocanegra R, Patterson TF. Comparison of lateral flow technology and galactomannan and (1->3)-beta-D-glucan assays for detection of invasive pulmonary aspergillosis. Clin Vaccine Immunol. 2009;16(12):1844-6. doi:10.1128/CVI.00268-09.

24. Marr KA, Laverdiere M, Gugel A, Leisenring W. Antifungal therapy decreases sensitivity of the Aspergillus galactomannan enzyme immunoassay. Clin Infect Dis. 2005;40(12):1762-9. doi:10.1086/ 429921.

25. Held J, Schmidt T, Thornton CR, Kotter E, Bertz H. Comparison of a novel Aspergillus lateral-flow device and the Platelia(R) galactomannan assay for the diagnosis of invasive aspergillosis following haematopoietic stem cell transplantation. Infection. 2013;41(6):1163-9. doi:10.1007/s15010-013-0472-5.

26. White PL, Parr C, Thornton C, Barnes RA. Evaluation of real-time PCR, galactomannan enzyme-linked immunosorbent assay (ELISA), and a novel lateral-flow device for diagnosis of invasive aspergillosis. J Clin Microbiol. 2013;51(5):1510-6. doi:10.1128/ JCM.03189-12.

27. Willinger B, Lackner M, Lass-Florl C, Prattes J, Posch V, Selitsch $\mathrm{B}$, et al. Bronchoalveolar lavage lateral-flow device test for invasive pulmonary aspergillosis in solid organ transplant patients: a semiprospective multicenter study. Transplantation. 2014. doi:10. 1097/TP.0000000000000153.

28. Eigl S, Prattes J, Lackner M, Willinger B, Spiess B, Reinwald M, et al. Multicenter evaluation of a lateral-flow device test for diagnosing invasive pulmonary aspergillosis in ICU patients. Crit Care. 2015;19(1):178. doi:10.1186/s13054-015-0905-x.

29. Azoulay E, Dupont H, Tabah A, Lortholary O, Stahl JP, Francais A, et al. Systemic antifungal therapy in critically ill patients without invasive fungal infection*. Crit Care Med. 2012;40(3):813-22. doi: 10.1097/CCM.0b013e318236f297.

30. Johnson G, Shannon M, Thornton C, Agrawal S, Lass-Floerl C, Mutschlechner W, et al. Proximity ligation assay for the early detection of invasive aspergillosis. 25th European Congress of Clinical Microbriology and Infectious Diseases; 25 - 28 April 2015. Copenhagen; 2015.

31. Ong EL. Common AIDS-associated opportunistic infections. Clin Med. 2008;8(5):539-43.

32. Park BJ, Wannemuehler KA, Marston BJ, Govender N, Pappas PG, Chiller TM. Estimation of the current global burden of cryptococcal meningitis among persons living with HIV/AIDS. AIDS. 2009;23(4):525-30. doi:10.1097/QAD.0b013e328322ffac.

33. Mirza SA, Phelan M, Rimland D, Graviss E, Hamill R, Brandt ME, et al. The changing epidemiology of cryptococcosis: an update from population-based active surveillance in 2 large metropolitan areas, 1992-2000. Clin Infect Dis. 2003;36(6):789-94. doi:10.1086/368091.

34. Makadzange AT, Ndhlovu CE, Takarinda K, Reid M, Kurangwa M, Gona $\mathrm{P}$, et al. Early versus delayed initiation of antiretroviral therapy for concurrent HIV infection and cryptococcal meningitis in sub-Saharan Africa. Clin Infect Dis. 2010;50(11):1532-8. doi:10. 1086/652652.

35. Lessells RJ, Mutevedzi PC, Heller T, Newell ML. Poor long-term outcomes for cryptococcal meningitis in rural South Africa. S Afr Med J = Suid-Afrikaanse tydskrif vir geneeskunde. 2011;101(4): 251-2.

36. Babady NE, Bestrom JE, Jespersen DJ, Jones MF, Beito EM, Binnicker MJ, et al. Evaluation of three commercial latex agglutination kits and a commercial enzyme immunoassay for the detection of cryptococcal antigen. Med Mycol. 2009;47(3):336-8. doi: 10.1080/13693780802607400.

37. Hamilton JR, Noble A, Denning DW, Stevens DA. Performance of cryptococcus antigen latex agglutination kits on serum and cerebrospinal fluid specimens of AIDS patients before and after pronase treatment. J Clin Microbiol. 1991;29(2):333-9.

38. Anderson DA, Crowe SM, Garcia M. Point-of-care testing. Curr HIV/AIDS Rep. 2011;8(1):31-7. doi:10.1007/s11904-010-0067-z.

39. Vijayan T, Chiller T, Klausner JD. Sensitivity and specificity of a new cryptococcal antigen lateral flow assay in serum and cerebrospinal fluid. MLO Med Lab Obs. 2013;45(3):16. 8, 20.

40. Kozel TR, Bauman SK. CrAg lateral flow assay for cryptococcosis. Expert Opin Med Diagn. 2012;6(3):245-51. doi:10.1517/ 17530059.2012.681300.

41. Binnicker MJ, Jespersen DJ, Bestrom JE, Rollins LO. Comparison of four assays for the detection of cryptococcal antigen. Clin Vaccine Immunol. 2012;19(12):1988-90. doi:10.1128/CVI. 00446-12.

42. McMullan BJ, Halliday C, Sorrell TC, Judd D, Sleiman S, Marriott $\mathrm{D}$, et al. Clinical utility of the cryptococcal antigen lateral flow assay in a diagnostic mycology laboratory. PLoS One. 2012;7(11):e49541. doi:10.1371/journal.pone.0049541.

43. Escandon P, Lizarazo J, Agudelo CI, Chiller T, Castaneda E. Evaluation of a rapid lateral flow immunoassay for the detection of cryptococcal antigen for the early diagnosis of cryptococcosis in HIV patients in Colombia. Med Mycol. 2013;51(7):765-8. doi:10. 3109/13693786.2013.781692.

44. Lindsley MD, Mekha N, Baggett HC, Surinthong Y, Autthateinchai $\mathrm{R}$, Sawatwong P, et al. Evaluation of a newly developed lateral flow immunoassay for the diagnosis of cryptococcosis. Clin Infect Dis. 2011:53(4):321-5. doi:10.1093/cid/cir379.

45. Magambo KA, Kalluvya SE, Kapoor SW, Seni J, Chofle AA, Fitzgerald DW, et al. Utility of urine and serum lateral flow assays to determine the prevalence and predictors of cryptococcal antigenemia in HIV-positive outpatients beginning antiretroviral therapy in Mwanza, Tanzania. J Int AIDS Soc. 2014;17:19040. doi:10.7448/IAS.17.1.19040.

46. Boulware DR, Rolfes MA, Rajasingham R, von Hohenberg M, Qin $\mathrm{Z}$, Taseera $\mathrm{K}$, et al. Multisite validation of cryptococcal antigen lateral flow assay and quantification by laser thermal contrast. Emerg Infect Dis. 2014;20(1):45-53. doi:10.3201/eid2001.130906.

47. Kabanda T, Siedner MJ, Klausner JD, Muzoora C, Boulware DR. Point-of-care diagnosis and prognostication of cryptococcal meningitis with the cryptococcal antigen lateral flow assay on cerebrospinal fluid. Clin Infect Dis. 2014;58(1):113-6. doi:10.1093/cid/ cit641. 
48. Jarvis JN, Percival A, Bauman S, Pelfrey J, Meintjes G, Williams GN, et al. Evaluation of a novel point-of-care cryptococcal antigen test on serum, plasma, and urine from patients with HIV-associated cryptococcal meningitis. Clin Infect Dis. 2011;53(10):1019-23. doi:10.1093/cid/cir613.

49. World Health Organisation. Rapid advice: Diagnosis, prevention and management of cryptococcal disease in HIV-infected adults, adolescents and children. 2011; p. 44.

50. Grinsell M, Weinhold LC, Cutler JE, Han Y, Kozel TR. In vivo clearance of glucuronoxylomannan, the major capsular polysaccharide of Cryptococcus neoformans: a critical role for tissue macrophages. J Infect Dis. 2001;184(4):479-87. doi:10.1086/322787.

51. Brouwer AE, Teparrukkul P, Pinpraphaporn S, Larsen RA, Chierakul W, Peacock S, et al. Baseline correlation and comparative kinetics of cerebrospinal fluid colony-forming unit counts and antigen titers in cryptococcal meningitis. J Infect Dis. 2005;192(4): 681-4. doi:10.1086/432073.

52. Aberg JA, Watson J, Segal M, Chang LW. Clinical utility of monitoring serum cryptococcal antigen (sCRAG) titers in patients with AIDS-related cryptococcal disease. HIV Clin Trials. 2000;1(1):1-6. doi:10.1310/NQXR-ULMG-MM1B-3T2B.

53. Antinori S, Radice A, Galimberti L, Magni C, Fasan M, Parravicini $\mathrm{C}$. The role of cryptococcal antigen assay in diagnosis and monitoring of cryptococcal meningitis. J Clin Microbiol. 2005;43(11): 5828-9. doi:10.1128/JCM.43.11.5828-5829.2005.

54. Bicanic T, Wood R, Meintjes G, Rebe K, Brouwer A, Loyse A, et al. High-dose amphotericin B with flucytosine for the treatment of cryptococcal meningitis in HIV-infected patients: a randomized trial. Clin Infect Dis. 2008;47(1):123-30. doi:10.1086/588792.

55. Liechty CA, Solberg P, Were W, Ekwaru JP, Ransom RL, Weidle $\mathrm{PJ}$, et al. Asymptomatic serum cryptococcal antigenemia and early mortality during antiretroviral therapy in rural Uganda. Tropical Med Int Health. 2007;12(8):929-35. doi:10.1111/j.1365-3156. 2007.01874.x.

56. Worodria W, Massinga-Loembe M, Mazakpwe D, Luzinda K, Menten J, Van Leth F, et al. Incidence and predictors of mortality and the effect of tuberculosis immune reconstitution inflammatory syndrome in a cohort of TB/HIV patients commencing antiretroviral therapy. J Acquir Immune Defic Syndr. 2011;58(1):32-7. doi: 10.1097/QAI.0b013e3182255dc2.

57. Jarvis JN, Lawn SD, Vogt M, Bangani N, Wood R, Harrison TS. Screening for cryptococcal antigenemia in patients accessing an antiretroviral treatment program in South Africa. Clin Infect Dis. 2009;48(7):856-62. doi:10.1086/597262.
58. Lawn SD, Myer L, Harling G, Orrell C, Bekker LG, Wood R. Determinants of mortality and nondeath losses from an antiretroviral treatment service in South Africa: implications for program evaluation. Clin Infect Dis. 2006;43(6):770-6. doi:10.1086/ 507095.

59. French N, Gray K, Watera C, Nakiyingi J, Lugada E, Moore M, et al. Cryptococcal infection in a cohort of HIV-1-infected Ugandan adults. AIDS. 2002;16(7):1031-8.

60. Jarvis JN, Harrison TS, Govender N, Lawn SD, Longley N, Bicanic $\mathrm{T}$, et al. Routine cryptococcal antigen screening for HIV-infected patients with low CD4+ T-lymphocyte counts-time to implement in South Africa? S Afr Med J = Suid-Afrikaanse tydskrif vir geneeskunde. 2011;101(4):232-4.

61. Micol R, Tajahmady A, Lortholary O, Balkan S, Quillet C, Dousset JP, et al. Cost-effectiveness of primary prophylaxis of AIDS associated cryptococcosis in Cambodia. PLoS One. 2010;5(11):e13856. doi:10.1371/journal.pone.0013856.

62. Meya DB, Manabe YC, Castelnuovo B, Cook BA, Elbireer AM, Kambugu A, et al. Cost-effectiveness of serum cryptococcal antigen screening to prevent deaths among HIV-infected persons with a CD $4+$ cell count $<$ or $=100$ cells $/$ microL who start HIV therapy in resource-limited settings. Clin Infect Dis. 2010;51(4):448-55. doi: $10.1086 / 655143$.

63. Hoenigl M, Schwetz I, Wurm R, Scheidl S, Olschewski H, Krause R. Pulmonary histoplasmosis in three Austrian travelers after a journey to Mexico. Infection. 2008;36(3):282-4. doi:10.1007/ s15010-007-6298-2.

64. Scheel CM, Zhou Y, Theodoro RC, Abrams B, Balajee SA, Litvintseva AP. Development of a loop-mediated isothermal amplification method for detection of Histoplasma capsulatum DNA in clinical samples. J Clin Microbiol. 2014;52(2):483-8. doi:10.1128/ JCM.02739-13.

65. Wheat LJ, Freifeld AG, Kleiman MB, Baddley JW, McKinsey DS, Loyd JE, et al. Clinical practice guidelines for the management of patients with histoplasmosis: 2007 update by the Infectious Diseases Society of America. Clin Infect Dis. 2007;45(7):807-25. doi:10.1086/521259.

66. Hoenigl M, Prattes J, Spiess B, Wagner J, Prueller F, Raggam $\mathrm{RB}$, et al. Performance of galactomannan, beta-D-glucan, aspergillus lateral-flow device, conventional culture and PCR tests for diagnosis of invasive pulmonary aspergillosis in bronchoalveolar lavage fluid. J Clin Microbiol. 2014. doi:10.1128/ JCM.00467-14. 